\title{
Anti-acne effects of Castanea crenata bur extract and identification of active compound
}

\author{
Jiyoung You ${ }^{1}$, Hyanggi Ji', Kyung-Baeg Roh' ${ }^{1}$ Eunae Cho ${ }^{1}$, Hanane Chajra ${ }^{2}$, Mathilde Frechet ${ }^{2}$, \\ Deokhoon Park ${ }^{1}$ and Eunsun Jung ${ }^{1 *}$ (D)
}

\begin{abstract}
Acne vulgaris is a common disease of the pilosebaceous unit. Hyperseborrhea, a follicular colonization by Cutibacterium acnes and a complex inflammatory state are pathogenic factors of acne vulgaris. In the present study we investigated the anti-acne efficacy of Castanea crenata bur extract (CBE) in vitro and searched active compound for mitigating hyperseborrhea. In sebocytes, CBE inhibited the sebum synthesis through downregulation of sterol response element-binding protein-1 and peroxisome proliferator-activated receptor $\gamma$ expression. CBE also inhibited the 5-alpha reductase activity which is associated with androgen-induced sebum production. Moreover, CBE showed anti-inflammatory effect in C. acnes and free fatty acid-induced inflammatory condition through suppressing Toll-like receptor 2 activity. Anti-inflammatory effect was also observed in keratinocytes via inhibition of NF-kB translocation into nuclei. Finally, we identified the ellagic acid as an active compound for inhibiting sebum production in CBE. These findings suggest that CBE have potential to be a multi-target agent for acne vulgaris and a good source of ellagic acid as an anti-sebum compound.
\end{abstract}

Keywords: Castanea crenata, Sebocyte, Sebum synthesis, Anti-inflammation, 5-alpha reductase

\section{Introduction}

Acne vulgaris is a chronic inflammatory skin disease related to the pilosebaceous unit. In acne lesions, hyperseborrhea, dysseborrhea, elevated dihydrotestosterone (DHT) levels, abnormal follicular keratinization responsible for the comedone formation, an imbalance in skin microflora, particularly an increase of Cutibacterium acnes (C. acnes) colonization, and an inflammation have been observed [1].

Sebum is produced by sebocytes which constitute a major part of the sebaceous glands (SGs). Sebocytes start proliferation at the junctional zone of SGs and hair follicles (HFs) and differentiation begin after migration to the maturation zone of the SGs. Fully differentiated sebocytes secrete sebum through a lysis process, which called

\footnotetext{
${ }^{*}$ Correspondence: bioso@biospectrum.com

${ }^{1}$ Biospectrum Life Science Institute, Suji-Gu, Yongin, Gyeonggi-Do 16827,

Republic of Korea

Full list of author information is available at the end of the article
}

a holocrine secretion. Sebum production is affected by steroid hormones, vitamin A derivatives, insulin-like growth factor 1 (IGF-1), and free fatty acids (FFAs). Sterol response element-binding protein-1 (SREBP-1) and peroxisome proliferator-activated receptors (PPARs) are key transcriptional regulators of lipid synthesis in sebocytes [2].

Sebum is a mixture of lipids mainly composed of triglyceride (TG) and FFAs [3]. TG is hydrolyzed into FFAs by bacteria, especially $C$. acnes, as well as genuine FFAs secreted from sebocytes. Excessive sebum production is a major event in puberty acne, which contributes to comedone formation, sebogenesis, and inflammation [4]. Palmitic acid (PA), saturated fatty acid, is recently discovered to play an important role in upregulation of lipid contents and inflammatory response of the skin [5].

Higher androgen level is known to contribute to acne vulgaris through activation of sebum production. Testosterone, the major sex hormone in men, is converted to DHT by $5 \alpha$-reductase. DHT is an agonist of 
the androgen receptor (AR) with about two-to four-fold higher affinity than testosterone. Binding with AR in the cytoplasm lead to translocate AR into the nucleus and then subsequently activates the lipid metabolism related gene expression. Thus, the downregulation of $5 \alpha$-reductase has been considered as a target for acne vulgaris by inhibiting excessive sebum production [6].

During acne lesion development, inflammation is observed at most stages. C. acnes is one of the major bacteria responsible for acne and plays a critical role in induction and maintenances of inflammation. In keratinocytes, C. acnes is reported to activate inflammation through Toll-like receptor 2 (TLR2) and nuclear factor kappa-light-chain-enhancer of activated B (NF$\kappa B)$ pathways [7]. Along with the inhibition of sebum production in sebocytes, the downregulation of inflammation induced by $C$. acnes is an additional therapeutic alternative to remedy and prevents acne vulgaris.

Castanea crenata Siebold and Zucc (C. crenata) is classified in the Fagaceae family and inhabits northeast asian countries including Korea and Japan. The extracts from various parts of this species have been reported to exert antioxidant, antimicrobial, and anti-allergic activities [8]. C. crenata bark contains tannin used as preservatives, the outer and inner shells of $C$. crenata have anti-adipogenic activity, and other parts like leaves, flowers, and the inner skin abound with phenolic and flavonoid compounds with antioxidant properties [9]. C. crenata bur (chestnut bur), also known as involucre, is a waste product of chestnuts and has been used as traditional medicine for erysipelas, pertussis, and bronchitis. The anti-viral activity of the flavonoid glycoside derived from chestnut bur was reported by Kim et al. [10], but the effects on the skin have not been elucidated yet. In this study, we investigated the antiacne properties of $C$. crenata bur extract on the three well described acne triggers: hyperseborrhea (sebum production), androgenic hormone level ( $5 \alpha$-reductase activity), and inflammation induced by $C$. acnes and FFAs, and then elucidated the active compound for inhibiting sebum synthesis.

\section{Materials and methods}

\section{Preparation of $C$. crenata bur extract}

Dried C. crenata bur purchased from the Pungcheon Ginseng Medicinal Herbs Farming union (Korea, $33 \mathrm{~g}$ ) was extracted with distilled water $(320 \mathrm{~mL})$ for $3 \mathrm{~h}$ at $100{ }^{\circ} \mathrm{C}$ and filtered. The extract was concentrated in a rotary evaporator at $55{ }^{\circ} \mathrm{C}$ and dried using a freeze dryer at $50{ }^{\circ} \mathrm{C}$ for $24 \mathrm{~h}$. The yield of C. crenata bur extract is $2.8 \%$. The extract dissolved in distilled water was named C. crenata bur extract (CBE) and used in all experiments.

\section{Sebocytes culture and Nile red staining for lipid synthesis} Human sebocytes purchased from Celprogen (Torrance, CA, USA) were cultured in Human Sebocyte Complete Growth Media (Celprogen) at $37{ }^{\circ} \mathrm{C}$ in a $5 \% \mathrm{CO}_{2}$ incubator. Human sebocytes were differentiated with $100 \mu \mathrm{M}$ palmitic acid (Sigma-Aldrich, St. Louis, MO, USA) with or without CBE or ellagic acid for $48 \mathrm{~h}$. The treated cells were washed using $1 \times \mathrm{PBS}$ and fixed with $4 \%$ formaldehyde for $10 \mathrm{~min}$ at room temperature (RT). After washing three times, the cells were stained with $10 \mu \mathrm{g} / \mathrm{mL}$ Nile red (Sigma-Aldrich) diluted in $1 \times$ PBS at a 1:100 ratio for $15 \mathrm{~min}$ at RT. The nuclei were stained with Hoechst 33342 (Thermo Fisher Scientific, Waltham, MA, USA) solution and fluorescence was detected at excitation and emission wavelengths of $550 \mathrm{~nm}$ and $630 \mathrm{~nm}$, respectively.

\section{Triglyceride (TG) ELISA assay}

TG released from sebocytes were measured by using ELISA (Triglyceride Quantification Assay Kit, Abcam, Cambridge, UK) according to the manufacturer's instructions. The reaction mixture was added and shaken for $60 \mathrm{~min}$ at RT protected from light and the absorbance was measured at $570 \mathrm{~nm}$ using a spectrophotometer (BioTek, Winooski, VT, USA).

\section{Western blot analysis of SREBP-1 and PPAR $\gamma$}

For western blot analysis of SREBP-1 and PPAR $\gamma$, the sebocytes were seeded into a $60 \mathrm{~mm}$ dish and incubated in Dulbecco's modified Eagle's medium (DMEM, Hyclone, Logan, UT, USA) containing $1 \%$ serum and treated with $\mathrm{CBE}$ at 1,10 and $50 \mu \mathrm{g} / \mathrm{mL}$ at $37^{\circ} \mathrm{C}$ for 3 days. Cells were collected from the dishes and lysed with lysis buffer (PRO-PREP, iNtRON Biotechnology, Seongnam, Korea). After sonication, the cell lysate was centrifuged at $16,200 \times g$ for $10 \mathrm{~min}$ and the pellet was removed. Proteins were quantified by the Bradford assay and separated by sodium dodecyl sulfate-polyacrylamide gel electrophoresis (SDS-PAGE). The proteins were transferred to nitrocellulose membranes using a transfer kit (iBlot2, iBlot gel transfer stacks, Thermo Fisher Scientific) and blocked with $5 \%$ skim milk solubilized in $1 \times$ PBS containing $0.1 \%$ Tween 20 (PBST, Sigma-Aldrich) at RT for $1 \mathrm{~h}$. Then, the membranes were incubated with PPAR $\gamma$ antibody (1:1000) (Cell Signaling Technology, Danvers, MA, USA), SREBP1 antibody (1:1000) (Novus Biologicals, Centennial, CO, USA), and GAPDH (1:1000) (Santa Cruz Biotechnology, Inc., Dallas, TX, USA) at $4{ }^{\circ} \mathrm{C}$ overnight. After washing with PBST three times, the membrane was incubated with mouse or rabbit IgG secondary antibody (1:1000, Cell Signaling Technology) at RT for $1 \mathrm{~h}$ and the reaction was developed with 
chemiluminescence substrate (GloBrite ECL Reagent Kit, R\&D System, Detroit, MI, USA). The detected proteins were normalized to the individual non-phosphorylated form or GAPDH.

\section{5a-reductase activity assay}

Active solution $(0.5 \mathrm{~mL})$ was prepared with or without a CBE in $1 \mathrm{mmol} / \mathrm{L}$ dithiothreitol (Thermo Fisher Scientific), $\mathrm{pH} 6.5,40 \mathrm{mmol} / \mathrm{L}$ potassium phosphate (SigmaAldrich), $100 \mu \mathrm{mol} / \mathrm{L}$ NADPH (Roche Holding AG, IN, USA), and $3.5 \mu \mathrm{mol} / \mathrm{L}$ testosterone (ChemFaces, Hubei, China) for $20 \mathrm{~min}$ at RT. Then, $20 \mu \mathrm{g}$ of rat liver microsomes (Sigma-Aldrich) was added and incubated for $30 \mathrm{~min}$ at $37^{\circ} \mathrm{C}$ to start the reaction. After lyophilizing the reaction solution, it was dissolved in methanol and filtered through a 0.2-micron filter (Pall, NY, USA). Finally, testosterone was measured by HPLC (Waters 2695 Separation Module, Waters 2996 Photodiode Array Detector, Waters, MA, USA) on a C18 column $(4.6 \times 150 \mathrm{~mm}$, $2.6 \mu \mathrm{m}$, Phenomenex, Torrance, CA, USA) at RT, and a UV detection wavelength of $245 \mathrm{~nm}$. The analysis was conducted with an isocratic mobile phase of $0.1 \%$ trifluoroacetic acid in water: acetonitrile $(50: 50, \mathrm{v} / \mathrm{v})$ at a flow rate of $1.0 \mathrm{~mL} / \mathrm{min}$.

\section{Preparation of the conditioned medium of $C$. acnes}

The strain of $C$. acnes ATCC 6919 used in this study was obtained from the American Type Culture Collection (ATCC, Manassas, VA, USA). C. acnes was anaerobically cultured in Reinforced Clostridial Medium (RCM, Difco, Franklin Lakes, NJ, USA) at $37{ }^{\circ} \mathrm{C}$ for 48 h. C. acnes was cultured in a 96-well microplate with or without CBE, respectively. C. acnes was adjusted to $1 \times 10^{7}$ colonyforming units $(\mathrm{CFU}) / \mathrm{mL}$ in RCM. CBE was dissolved in distilled water and prepared in media at concentrations of $10,50,100,500,1000$, and $2000 \mu \mathrm{g} / \mathrm{mL}$. Each well was inoculated with $1 \times 10^{5} \mathrm{CFU} / \mathrm{mL}$ of the bacterial suspension and the microplate was incubated for $18 \mathrm{~h}$. After collecting the conditioned medium, the supernatant was filtrated through a 0.22-micron pore-sized filter (EMD Millipore, Temecula, CA, USA) and treated with 1/100 dilution in cells. The conditioned medium recovered by culturing $C$. acnes was named $C$. acnes $\mathrm{CM}$, and the conditioned medium with $\mathrm{CBE}$ was named $C$. acnes $\mathrm{CBE}$, respectively (Fig. 1A, B).

\section{TLR2/NF-KB/SEAP activity assay}

HEK293-hTLR2 cells (HEK293-Blue ${ }^{\mathrm{TM}}$ hTLR2, InvivoGen, San Diego, CA, USA) was used to determine the effect of CBE on TLR2. The TLR2 reporter cell line is a stably co-transfected cell line which expresses fulllength human TLR2 and the secreted alkaline phosphatase (SEAP) reporter gene under the transcriptional

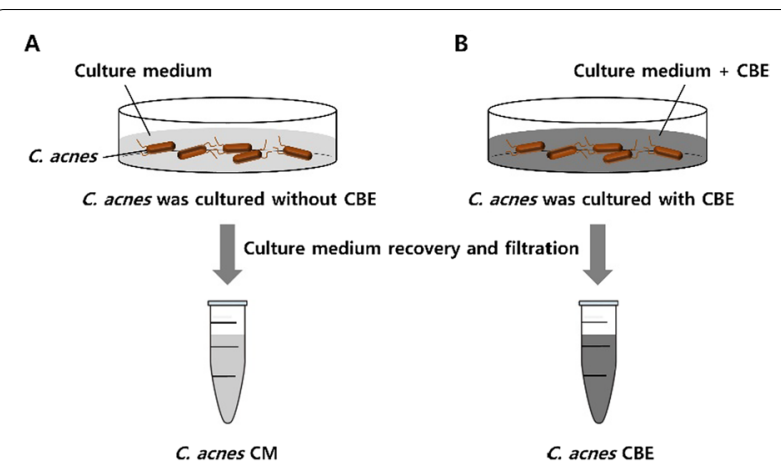

Fig. 1 Schematic of the preparation of the cultured medium of $C$. acnes. A C. acnes CM. B C. acnes CBE

control of an NF-KB response element. HEK293-hTLR2 cells were cultured in DMEM with $4 \mathrm{mM}$ L-glutamine, $4500 \mathrm{mg} / \mathrm{L}$ glucose, and sodium pyruvate supplemented with $10 \%$ fetal bovine serum (FBS, Gibco) at $37{ }^{\circ} \mathrm{C}$ in a $5 \% \mathrm{CO}_{2}$ incubator. HEK293-hTLR2 cells were seeded in 48-well plates and cultured in serumfree DMEM with $C$. acnes $\mathrm{CM}$ or $C$. acnes $\mathrm{CBE}$. After $6 \mathrm{~h}$, cultured medium was collected and reacted with QUANTI-Blue $^{\mathrm{TM}}$ solution (InvivoGen), then incubated at $37{ }^{\circ} \mathrm{C}$ in a $5 \% \mathrm{CO}_{2}$ incubator for $30 \mathrm{~min}$. SEAP activity was measured by reading the optical density (OD) at $620 \mathrm{~nm}$ with a microplate reader.

\section{Immunocytochemistry assay of NF-kB p65 nuclear translocation}

NF- $\mathrm{kB}$ p65 nuclear translocation was detected to confirm inflammatory process activation in the human epidermal keratinocytes (HEKn). HEKn (ATCC, Manassas, VA, USA) were cultured in EpiLife ${ }^{\mathrm{TM}}$ Medium with $60 \mu \mathrm{M}$ calcium (Gibco, Carlsbad, CA, USA) and Human Keratinocyte Growth Supplement (HKGS, Gibco) with CBE and C. acnes CM at $37{ }^{\circ} \mathrm{C}$ in a $5 \% \mathrm{CO}_{2}$ incubator for $1 \mathrm{~h}$. After washing with PBS, the cells were fixed with $4 \%$ formaldehyde (Sigma-Aldrich), washed using PBS three times, and permeabilized with $0.2 \%$ Triton X-100 (BIOSESANG, Seongnam, Korea). Then cells were washed three times and blocked with 5\% albumin (Albumin Fraction V, bioWORLD, Dublin, $\mathrm{OH}, \mathrm{USA}$ ) in PBS. The cells were treated with NF-kB p65 polyclonal antibody $(1: 200)$ (eBioscience ${ }^{\mathrm{TM}}$, Thermo Fisher Scientific) overnight at $4{ }^{\circ} \mathrm{C}$, then with rabbit IgG antibody tagged with Alexa Fluor 488 (1:200) (Merck, Darmstadt, Germany). The nuclei were stained with Hoechst 33342 (Thermo Fisher Scientific) before detecting NF- $\mathrm{kB}$ p 65 translocation using a fluorescence microscope $\left(\right.$ EVOS $^{\circledR}$ FL, Thermo Fisher Scientific). 


\section{Western blot analysis of phospho-ІкBa and IKB}

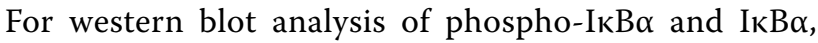
the HEKn were seeded into a $60 \mathrm{~mm}$ dish and incubated in DMEM containing 1\% FBS and treated with $\mathrm{CBE}$ at 1,10 and $50 \mu \mathrm{g} / \mathrm{mL}$ and $C$. acnes $\mathrm{CM}$ at $37^{\circ} \mathrm{C}$ for 3 days. Then, follow the same steps as above. The membranes were incubated with phospho-IкB $\alpha$ (Ser32) antibody (1:1000), IкB $\alpha$ antibody (1:1000) (Cell Signaling Technology), at $4{ }^{\circ} \mathrm{C}$ overnight.

\section{Activity guided identification of active compound}

C. crenata bur was extracted with boiling water in a reflux system. The aqueous extract $(10.15 \%)$ was suspended in $\mathrm{EtOH}(70 \%, \mathrm{v} / \mathrm{v})$, then the soluble part was evaporated and partitioned by chloroform, ethyl acetate, and $n$-butanol, depending upon the solvent polarity. Thus, the ethyl acetate fraction was further separated by methanol precipitation. After precipitation, the soluble part was separated using silica gel and Sephadex LH-20 column chromatography, and the precipitated fraction was dissolved in dimethyl sulfoxide (DMSO). Figure 6A shows the schematic of the fractionation of $\mathrm{CBE}$.

The HPLC system used in this study was a Waters 2695 (Waters, Milford, MA, USA) system equipped with a Waters 996 Photodiode Array (PDA) Detector. Empower 2 software was used to control the analytical system and perform the data collection and processing. HPLC-PDA analysis was performed on a Phenomenex Luna C18 (2) $(4.6 \times 150 \mathrm{~mm}, 5 \mu \mathrm{m})$ reverse-phase column protected by a C18 guard column from Phenomenex, Inc. (Torrance, CA, USA). The sample injection volume was $10 \mu \mathrm{L}$. The signal was monitored at $240 \mathrm{~nm}$. The elution system used for the HPLC-PDA assay was a binary high-pressure gradient elution system with mobile phase A $(0.1 \%$ trifluoroacetic acid (TFA) in $\mathrm{H}_{2} \mathrm{O}$ ) and mobile phase $\mathrm{B}$ (acetonitrile). The elution gradient was $10 \%$ organic phase $B$, held for 7 min; then, $10-55 \%$ organic phase B in 21 min (linear gradient); $55-70 \%$ organic phase B in 4 min (linear gradient); then return to the starting condition in $3 \mathrm{~min}$ and re-equilibration for $5 \mathrm{~min}$. The flow rate was $1.0 \mathrm{~mL} / \mathrm{min}$. Each analysis required $40 \mathrm{~min}$, including the re-equilibration time.

\section{Statistical analysis}

All experiments were conducted at least three times, and the data are expressed as the mean \pm standard deviation (SD). The data were analyzed by the student's $t$ test. A $P$ value less than 0.05 was considered statistically significant.

\section{Results}

Effect of CBE on sebum synthesis of sebocytes

Excessive sebum production known as hyperseborrhea is the major causative step of acne vulgaris. To confirm the inhibitory effect of $\mathrm{CBE}$ on sebum synthesis, we used a PA-induced differentiation model of sebocytes. PA has been reported to associated with sebum synthesis and inflammation in acne [3]. As shown in Fig. 2A, CBE significantly inhibited the PA-induced sebum production at a concentration of 10 and $50 \mu \mathrm{g} / \mathrm{mL}$. Since TG is a major component of sebum, we also quantified the secreted TG levels by using ELISA. As expected, CBE decreased the level of TG in a dose dependent manner (Fig. 2B). Then, to explore the regulatory mechanism of $\mathrm{CBE}$ on lipid synthesis, we assessed the expression level of SREBP-1 and PPAR $\gamma$, which are well-known transcriptional factors of lipid synthesis. As a result, we found that $\mathrm{CBE}$ decreased the expression levels of SREBP-1 and PPAR $\gamma$ induced by PA (Fig. 2C). These results suggest that CBE inhibited the PA-induced sebum production by downregulation of SREBP-1 and PPARy expression in sebocytes.

\section{Effect of CBE on 5a-reductase activity}

Androgen-induced sebum production is considerably related to $5 \alpha$-reductase enzyme activity, which converts testosterone to DHT. DHT, the active androgenic form of testosterone, has a higher affinity for the AR than testosterone. Therefore, the inhibition of $5 \alpha$-reductase can play a critical role in improving DHT-mediated acne through decreasing sebum synthesis. To explore the inhibitory effect of CBE on $5 \alpha$-reductase activity, we measured the remaining testosterone in an enzymatic reaction using testosterone and rat liver microsomes containing $5 \alpha$-reductase. The higher amount of testosterone in reaction mixture means that the activity of $5 \alpha$-reductase is inhibited. As shown in Fig. 3, CBE inhibited the conversion of testosterone to DHT in a dose dependent manner. Finasteride, used as a positive control, also showed inhibitory effect on $5 \alpha$-reductase. The result suggests that $\mathrm{CBE}$ can be effective to mitigate acne vulgaris by inhibition of $5 \alpha$-reductase activity.

\section{Effect of CBE on C. acnes and FFA-induced TLR2 activation in HEK293-hTLR2 cells}

TLR2 ligands present on $C$. acnes have been known to initiate inflammatory gene expression and contribute to the vicious inflammatory circle of acne. To explore the anti-inflammatory effect of CBE on TRL2 activity, we used the HEK293-hTLR2 cells in C. acnes and FFAs induced inflammation model. We first check whether CBE can inhibit the level of virulence factors secreted from $C$. acnes, we used the $C$. acnes $\mathrm{CM}$ and $C$. acnes $\mathrm{CBE}$. We applied the collected C. acnes $\mathrm{CM}$ and $C$. acnes 


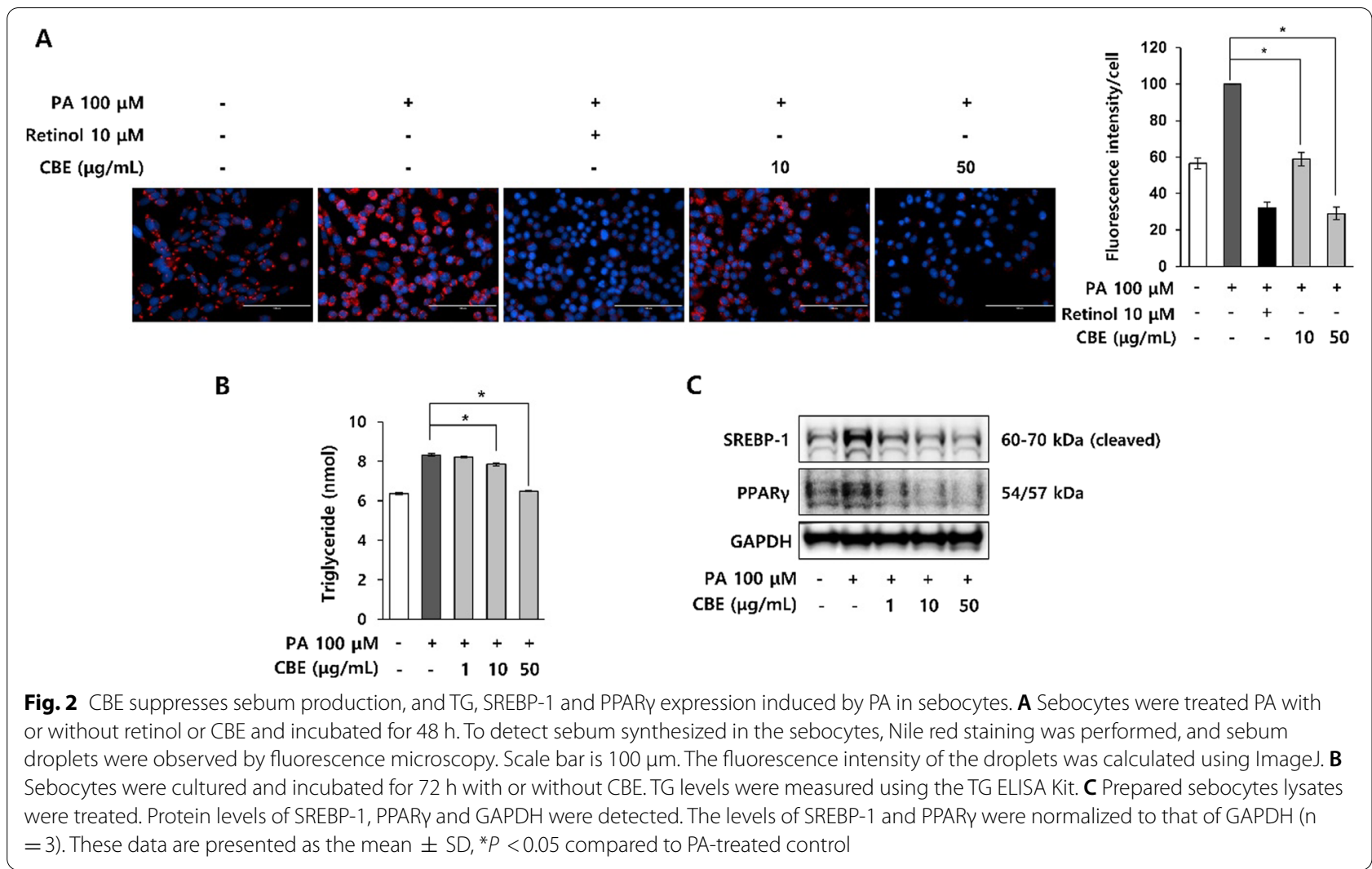

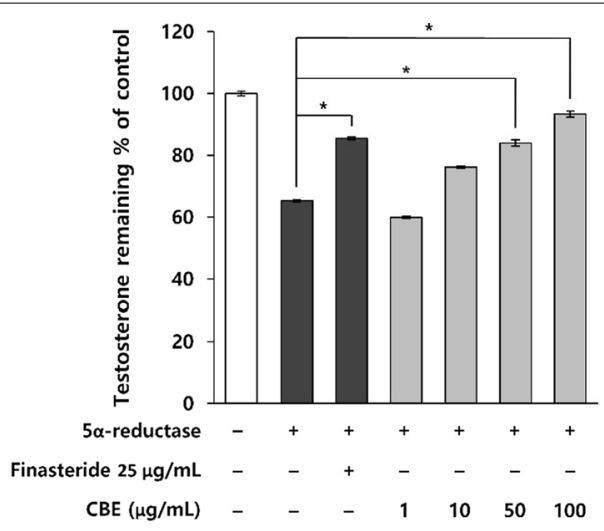

Fig. 3 CBE inhibits 5a-reductase activity. The liver microsomal fraction containing 5 a-reductase activity was put in contact to finasteride or CBE. After lyophilizing the reaction solution, the remaining testosterone was analyzed by HPLC $(n=3)$. The data are presented as the mean $\pm \mathrm{SD},{ }^{*} P<0.05$ compared to 5a-reductase-treated control

CBE to HEK293-hTLR2 cells for $6 \mathrm{~h}$ and then measured the TLR2 activity. As a result, we observed that $C$. acnes CBE significantly decreased the TLR2 activity compared to $C$. acnes CM (Fig. 4A). Then we found that CBE can attenuate the $C$. acnes $\mathrm{CM}$ induced inflammation in
HEK293-hTLR2 cells. As shown in Fig. 4B, CBE inhibited the $C$. acnes CM-induced TLR2 activation in a dose dependent manner. These results suggest that $\mathrm{CBE}$ can exert anti-inflammatory effect by inhibiting the secretion of virulence factors from $C$. acnes as well as direct action on $C$. acnes-induced inflammatory pathway.

PA, a FFA well-known to trigger inflammation in acne vulgaris, induces activation of TLR2 pathway and secreting IL-1 $\beta$ related to inflammasome $[5,11]$. We found that PA stimulates the TLR2 signaling pathway and this activation was effectively reduced by CBE (Fig. 4C). The result indicates that $\mathrm{CBE}$ can be also effective in inflammatory acne vulgaris lesions by preventing the inflammatory responses induced by PA. Therefore, it is expected that CBE can be effectively applied for relieving inflammation occurring in inflammatory acne vulgaris lesions.

\section{Effect of CBE on C. acnes-induced NF-KB activation in epidermal keratinocytes}

Keratinocytes are important in the innate immune activation of inflammatory acne lesions on the skin. To investigate whether CBE exert anti-inflammatory activity against $C$. acnes on HEKn, we performed an immunocytochemistry for NF- $\mathrm{kB}$ p65 translocation and western blot analysis for I $\mathrm{K} \mathrm{B} \alpha$ expression. As shown in Fig. 5A, upon treatment with $C$. acnes $\mathrm{CM}$, the nuclear 


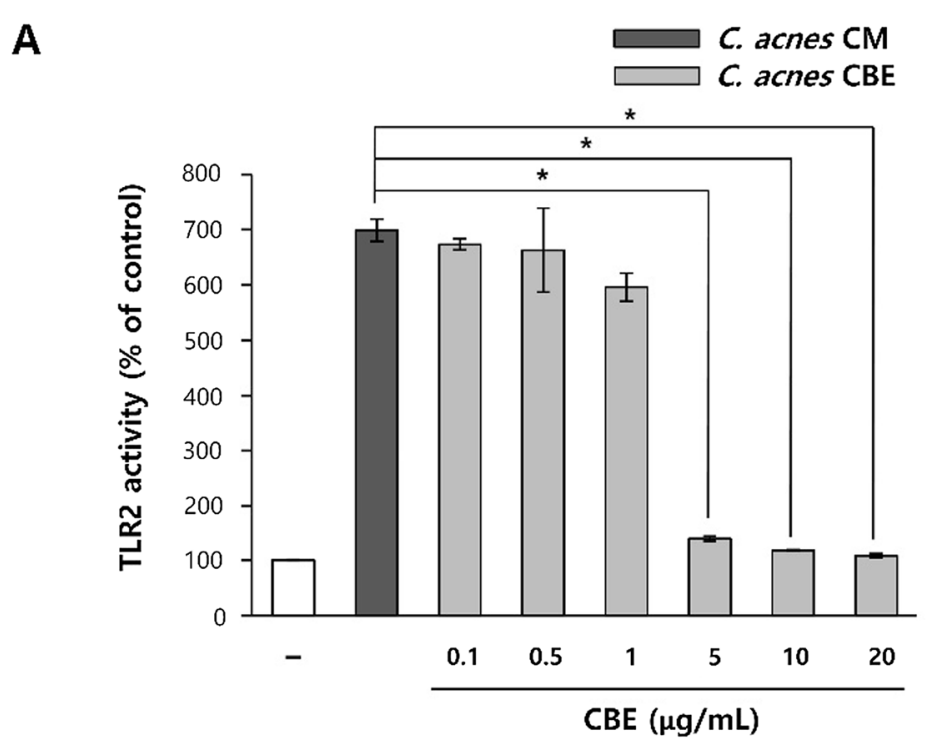

B

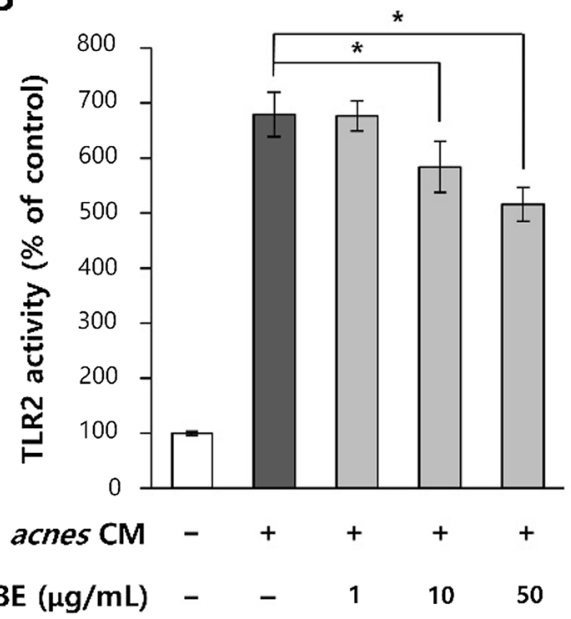

C

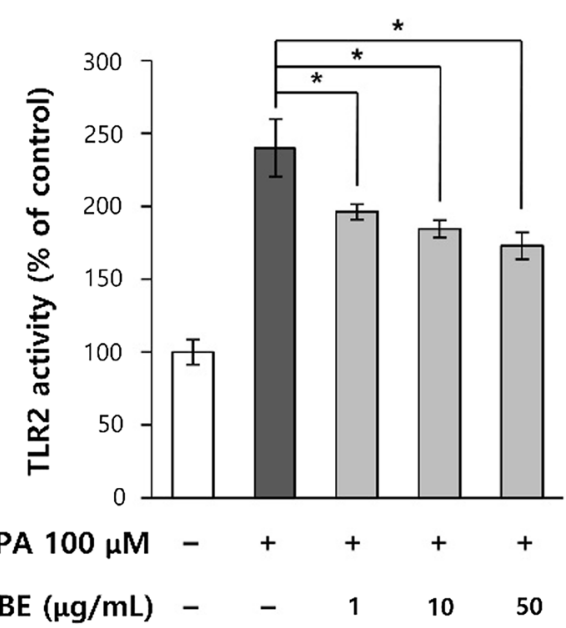

Fig. 4 CBE inhibits TLR2-mediated inflammatory pathway induced by C. acnes and PA in HEK293-TLR2 cells. A The HEK293-TLR2 cells were cultured in serum-free conditions and treated with C. acnes CM or C. acnes CBE. B The HEK293-TLR2 cells were cultured in serum-free conditions, treated with C. acnes CM, and CBE were added for 6 h. C The HEK293-TLR2 cells were cultured in serum-free conditions and PA was treated with or without CBE for $18 \mathrm{~h}$. TLR2 activity was measured through the secreted alkaline phosphatase. The values are expressed compared to the control, which was considered to be $100 \%(n=3)$. The data are presented as the mean $\pm S D, * P<0.05$ compared to the control group

translocation of NF-kB p65 was increased in keratinocytes, while treatment with $\mathrm{CBE}$ mitigated the $C$. acnes $\mathrm{CM}$-induced NF-kB p65 translocation. NF- $\mathrm{B}$ p 65 translocation is related to $\mathrm{I} \kappa \mathrm{B} \alpha$, which binds to NF- $\kappa \mathrm{B}$ and prohibits the translocation of NF- $\kappa B$ to the nucleus.

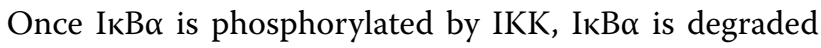
and the translocation of NF- $\mathrm{kB}$ p 65 occurs. Therefore,

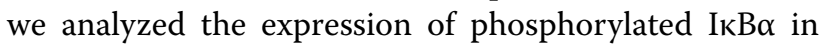
HEKn. In Fig. 5B, C. acnes CM increased the phosphorylation of IKB $\alpha$, which was inhibited by $C B E$ in a dose dependent manner. These data suggest that CBE suppress
C. acnes-induced inflammation by inhibiting NF- $\mathrm{KB}$ pathway in HEKn.

\section{Identification of ellagic acid as an active compound in CBE}

To elucidate the active compound of CBE responsible for sebum production, we performed a bioassay-guided fractionation (Fig. 6A). Among the fraction obtained, we found that the ethyl acetate fraction most effectively inhibits sebum production (Additional file 1: Figure S1). The inhibitory activity on sebum production was found in the precipitate (Additional file 1: Figure S2), and the 
A

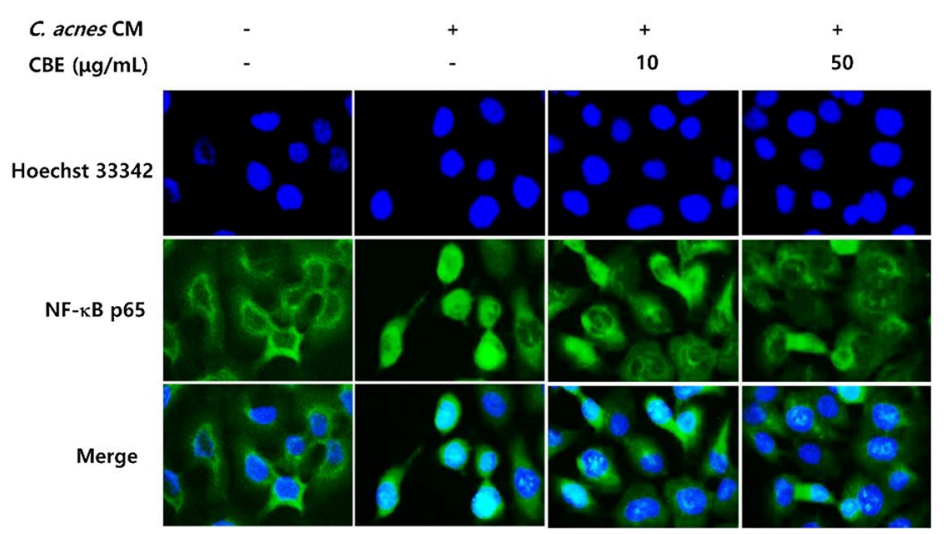

B

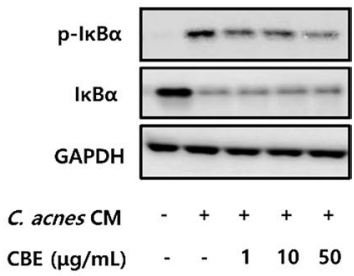

Fig. 5 CBE decreased C. acnes CM-induced NF-KB p65 translocation and IKBa phosphorylation in HEKn. A Immunofluorescence of NF-KB p65 subunit translocation in HEKn cultured in C. acnes CM or C. acnes CBE. The blue and green fluorescence indicates the nucleus and NF-KB p65 subunit, respectively. B After cell lysis, phosphorylated IKBa, IKBa, and GAPDH protein expression were detected by western blots. The protein levels were normalized to that of GAPDH, $n=3$

main active compound was determined to be ellagic acid using HPLC analysis (Fig. 6B). The content of ellagic acid was $1.1 \%$ in the aqueous extract, but it reached $64 \%$ in the fraction R10. To examine the effect of ellagic acid on sebocytes differentiation, sebocytes were treated with various concentrations $(1,10$, and $50 \mu \mathrm{g} / \mathrm{mL})$ of ellagic acid, and the level of lipid synthesis was analyzed by Nile red staining. Figure 7 shows that ellagic acid decreases the fluorescence intensity by $76 \%, 52 \%$, and $36 \%$ at 1,10 , and $50 \mu \mathrm{g} / \mathrm{mL}$, respectively, compared to the PA-treated control. These results demonstrate that ellagic acid is an active compound of $\mathrm{CBE}$ that inhibits the sebum accumulation induced by PA in sebocytes.

\section{Discussion}

Various pharmacological treatments have been considered as anti-acne therapies. Among them, retinoids have been widely used for a long time. Retinoids exert anti-acne efficacy through inhibition of $C$. acnesinduced cytokine secretion by reducing TLR2 pathway and downregulation of sebum production [12, 13]. Despite significant efficacy of retinoids on acne therapies, several adverse effects such as pruritus, erythema, peeling, burning sensation, cheilitis, xerosis, and local irritation has been consistently reported [14]. For this reason, it is very meaningful to develop safe anti-acne agents derived from natural materials that can replace retinoids. In this study, we demonstrated that $\mathrm{CBE}$ reduces lipid accumulation by decreasing the expression of SREBP-1 and PPAR $\gamma$, a regulator of fatty acid synthesis in sebocytes. And it was confirmed that EA from CBE was an active ingredient with an inhibitory effect on sebum synthesis. CBE also inhibited $5 \alpha$-reductase activity and suppressed the TLR-mediated inflammatory response leading to the activation of NF- $\mathrm{kB}$ induced by $C$. acnes and FFA. In addition, $\mathrm{CBE}$ downregulates the NF- $\mathrm{KB}$ translocation and $\mathrm{I} \kappa \mathrm{B} \alpha$ phosphorylation induced by $C$. acnes. Therefore, it is expected that $\mathrm{CBE}$ will have the ability to reduce comedolytic activity by reducing the NF- $\mathrm{kB}$ pathway in keratinocytes.

C. acnes is found in sebaceous glands producing sebum, which is used as the primary energy source of C. acnes. C. acnes-induced inflammation is an important step in various types of acne lesions by activating TLRs on keratinocytes and macrophages [15]. C. acnes secretes inflammatory factors called virulent molecules or factors like co-hemolytic Christie-Atkins-MunchPeterson (CAMP) factors, lipase, sialidases, hyaluronate lyase (HYL), and porphyrins, which are recognized by pattern recognition receptors. TLR2 recognizes pathogen-associated molecular patterns (PAMPs) secreted from $C$. acnes and activates NF- $\mathrm{kB}$ signalling, stimulating the transcription of pro-inflammatory cytokines in the nucleus [16]. Therefore, virulence factors are considered as major triggers of inflammation in acne. In this study, we indirectly observed the effect of $\mathrm{CBE}$ on the secretion of virulence factors from $C$. acnes by measuring TLR2 activity in $C$. acnes induced inflammation model. CBE inhibited TLR2 activity by inhibiting the secretion of virulence factors from $C$. acnes (Fig. 4A). Since CBE did not show any antimicrobial activity against $C$. acnes (Data not shown), the effect of CBE on TLR2 activation may derived from the 


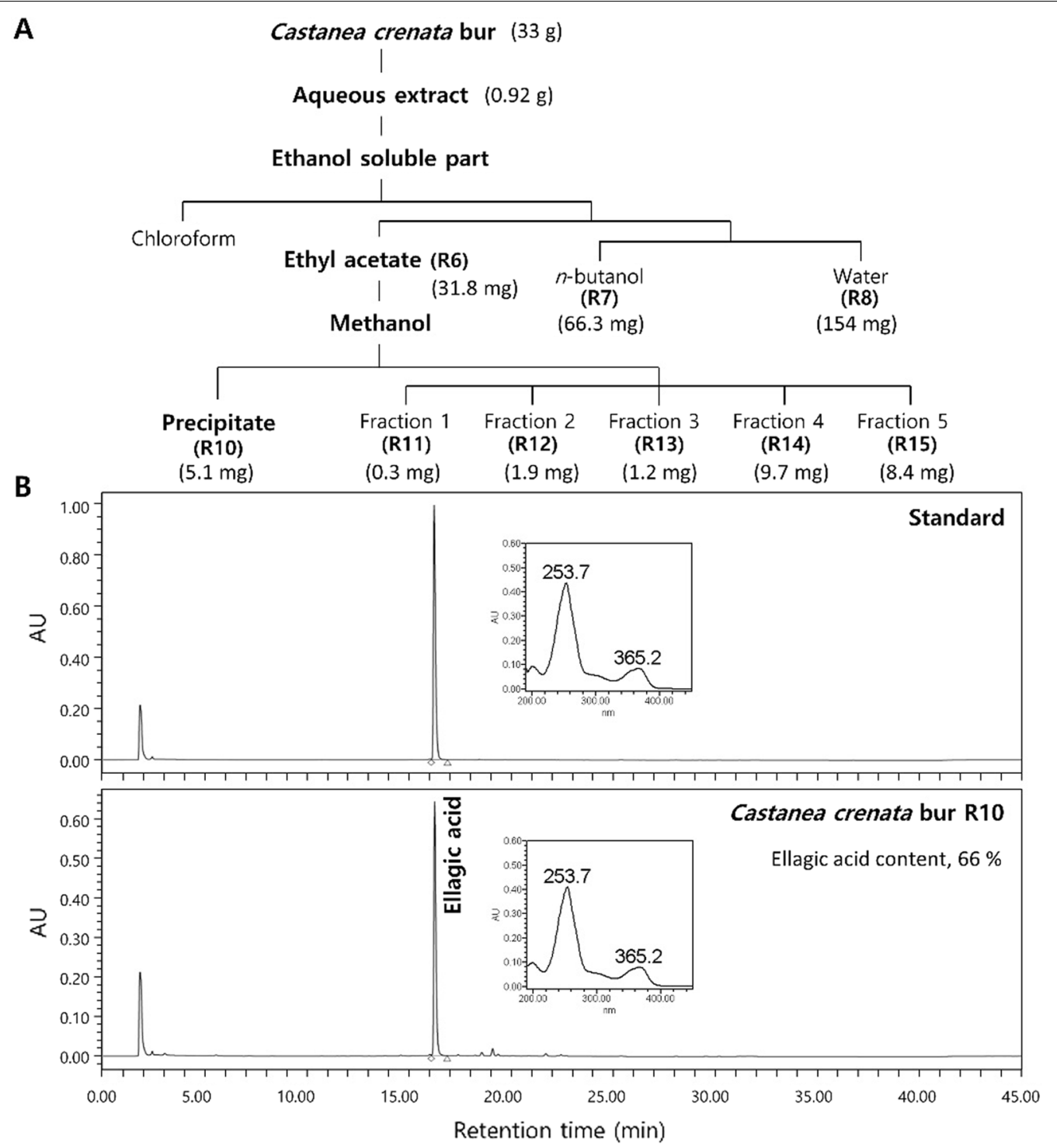

Fig. 6 CBE was fractionated and analyzed by HPLC. A Schematic of the fractionation of CBE. B Ellagic acid peak in the fraction R10 (lower) and in the ellagic acid standard (upper)

reduction of virulence factors secretion from $C$. acnes. Moreover, CBE directly inhibited the TLR2 activity induced by C. acnes CM (Fig. 4B).

In the epidermis, the keratinocytes inflammation through the activation of TLR2 is considered to have an important role in comedogenesis. PAMPs-induced TLR2 activation through NF-kB pathway stimulates normal human epidermal keratinocytes to release IL-1 $\alpha$, which promotes hypercornification like the TLR agonist-treated effect in isolated sebaceous glands [7]. Therefore, downregulating NF- $\mathrm{KB}$ activation in keratinocytes may contribute to reducing comedogenesis. In this study, we confirmed that $\mathrm{CBE}$ decreased $C$. acnes $\mathrm{CM}$-induced inflammation by inhibiting ІкB $\alpha$ phosphorylation, which subsequently inhibits NF- $\kappa$ B p65 translocation to nuclei in HEKn (Fig. 5). Therefore, CBE could reduce comedogenesis related to inflammation in acne vulgaris lesions by reducing TLR2 signaling through the NF-kB pathway.

Ellagic acid is a heterocyclic compound usually found in fruits and nuts of plant and has been reported to show anti-inflammatory, antioxidant, antiviral, anticancer and anti-adipogenic activity $[17,18]$. For example, ellagic acid reduces pro-inflammatory factors like TNF- $\alpha$, IL- 6 , and IL- $1 \beta$ in the sera of rats and recovers $\mathrm{D}$-galactose-reduced antioxidant activity by upregulating superoxide dismutase, catalase, and glutathione 

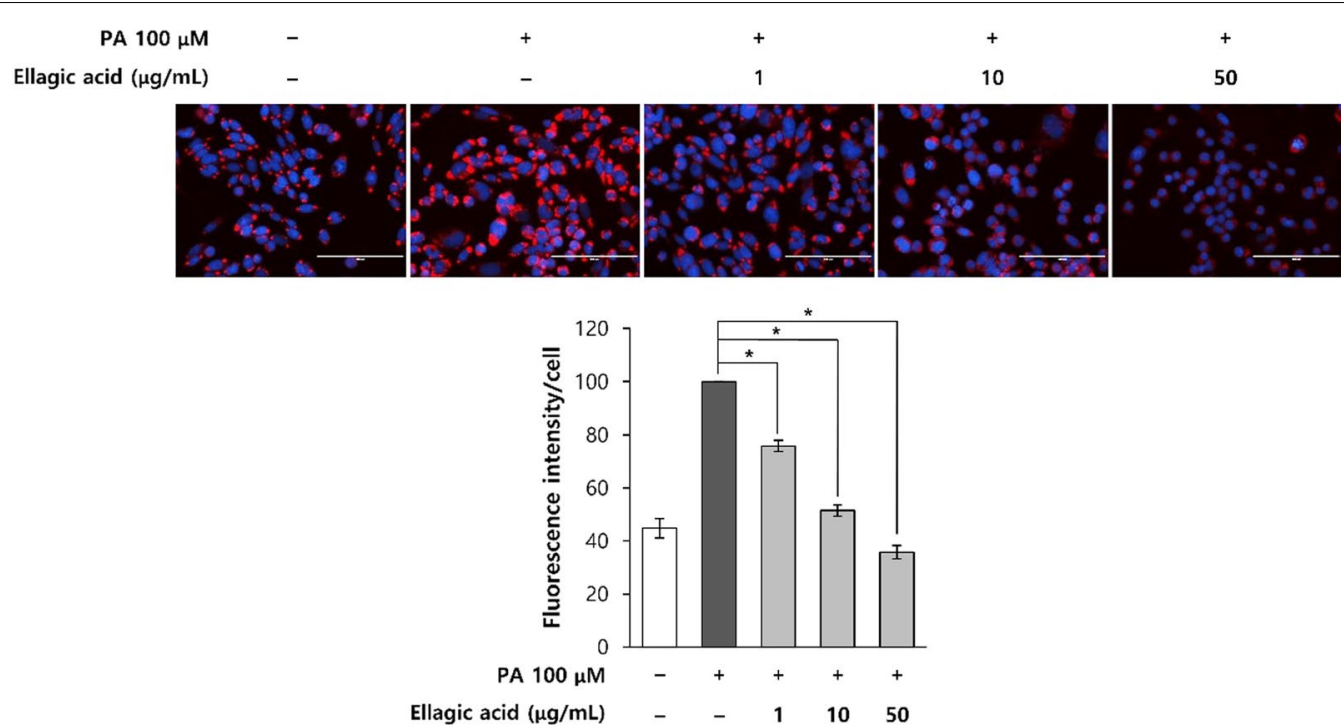

Fig. 7 Ellagic acid suppressed sebum production induced by PA in sebocytes. Sebocytes treated PA with or without ellagic acid and incubated for $48 \mathrm{~h}$. Sebum accumulation was detected by Nile red staining and sebum droplets were observed by fluorescence microscopy. Scale bar is $100 \mu \mathrm{m}$. The fluorescence intensity of the droplets was measured using ImageJ $(n=3)$. The data are presented as the mean $\pm S D, * P<0.05$ compared to the control group

peroxidase. It can also regulate cholesterol metabolism by suppressing SREBP-2 in rat livers as a bioactive compound of unripe Rubuscoreanus [19]. Recently, ellagic acid was shown to alleviate UVB-induced skin wrinkles by reducing phototoxicity and preventing collagen degradation [20]. However, its effects on acne have not yet been elucidated. In this study, we found that ellagic acid inhibits sebum accumulation in sebocytes. Further study is needed to confirm the efficacy of ellagic acid on the other target of acne and regulatory mechanism of sebum synthesis.

\section{Supplementary Information}

The online version contains supplementary material available at https://doi. org/10.1186/s13765-021-00670-x.

Additional file 1: Figure S1. Ethyl acetate fraction suppressed sebum production induced by PA in sebocytes. Sebocytes treated PA with or without fractions, ethyl acetate (R6), n-Butanol (R7) and water (R8) respectively, and incubated for $48 \mathrm{~h}$. Sebum accumulation was detected by Nile red staining and sebum droplets were observed by fluorescence microscopy. Scale bar is $100 \mu \mathrm{m}$. The fluorescence intensity of the droplets was measured using ImageJ $(n=3)$. The data are presented as the mean $\pm S D$, ${ }^{*} P<0.05$ compared to PA-untreated control; ${ }^{* *} P<0.05$ compared to PAtreated control. Figure $\mathbf{S 2}$. Precipitate of methanol extraction suppressed sebum production induced by PA in sebocytes. Sebocytes were treated with PA with or without several fractions, precipitate (R10), fraction 1 (R11), fraction 2 (R12), fraction 3 (R13), fraction 4 (R14) and fraction 5 (R15) for $48 \mathrm{~h}$. Sebum accumulation was detected by Nile red staining and sebum droplets were observed by fluorescence microscopy. Scale bar is $100 \mu \mathrm{m}$. The fluorescence intensity of the droplets was measured using ImageJ (n $=3$ ). The data are presented as the mean $\pm S D,{ }^{*} P<0.05$ compared to PA-untreated control; ${ }^{* *} P<0.05$ compared to PA-treated control. Figure S3. LC-MS/MS spectrum of the fraction R10.

\section{Authors' contributions}

$J Y$ and EJ: designed the experiments; JY: conducted the experiments; KBR, HJ and EC: assisted in experiments; JY and KR: analysis of data; JY, KR and EJ: wrote the manuscript; $\mathrm{HC}$ and MF: critical revision of manuscript. All authors have read and approved the final manuscript.

\section{Declarations}

\section{Competing interests}

The authors declare that they have no competing interests.

\section{Author details}

'Biospectrum Life Science Institute, Suji-Gu, Yongin, Gyeonggi-Do 16827, Republic of Korea. ${ }^{2}$ Clariant Production, 195 route d'Espagne, 31036 Toulouse, France.

Received: 19 October 2021 Accepted: 23 December 2021 Published online: 10 February 2022

\section{References}

1. Cong TX, Hao D, Wen X, Li XH, He G, Jiang X (2019) From pathogenesis of acne vulgaris to anti-acne agents. Arch Dermatol Res 311(5):337-349

2. Clayton RW, Göbel K, Niessen CM, Paus R, Steensel MAM, Lim X (2019) Homeostasis of the sebaceous gland and mechanisms of acne pathogenesis. Br J Dermatol 181(4):677-690

3. Lovászi M, Szegedi A, Zouboulis CC, Törőcsik D (2017) Sebaceousimmunobiology is orchestrated by sebum lipids. Dermato-Endocrinol 9(1):e1375636

4. Bergler-Czop B, Brzezińska-Wcisło L (2013) Dermatological problems of the puberty. Postepy Dermatol Alergol 30(3):178-187

5. Choi CW, Kim Y, Kim JE, Seo EY, Zouboulis CC, Kang JS, Youn SW, Chung $J H$ (2019) Enhancement of lipid content and inflammatory cytokine secretion in SZ95 sebocytes by palmitic acid suggests a potential link between free fatty acids and acne aggravation. Exp Dermatol 28(2):207-210 
6. Lai JJ, Chang P, Lai KP, Chen L, Chang C (2012) The role of androgen and androgen receptor in skin-related disorders. Arch Dermatol Res 304(7):499-510

7. Selway JL, Kurczab T, Kealey T, Langlands K (2013) Toll-like receptor 2 activation and comedogenesis: implications for the pathogenesis of acne. BMC Dermatol 13:10

8. Han SH, Hur MS, Kim MJ, Jung WH, Park M, Kim JH, Shin HJ, Choe YB, Ahn KJ, Lee YW (2017) In vitro anti-Malassezia activity of Castanea crenata shell and oil-soluble Glycyrrhiza extracts. Ann Dermatol 29(3):321-326

9. Youn UY, Shon MS, Kim GN, Katagiri R, Harata K, Ishida Y, Lee SC (2016) Antioxidant and anti-adipogenic activities of chestnut (Castanea crenata) byproducts. Food Sci Biotechnol 25(4):1169-1174

10. Kim N, Park S, Nhiem NX, Song JH, Ko HJ, Kim SH (2019) Cycloartanetype triterpenoid derivatives and a flavonoid glycoside from the burs of Castanea crenata. Phytochemistry 158:135-141

11. Snodgrass RG, Huang S, Choi IW, Rutledge JC, Hwang DH (2013) Inflammasome-mediated secretion of IL-1 $\beta$ in human monocytes through TLR2 activation; modulation by dietary fatty acids. J Immunol 191(8):4337-4347

12. Liu PT, Krutzik SR, Kim J, Modlin RL (2005) Cutting edge: all-trans retinoic acid down-regulates TLR2 expression and function. J Immunol 174(5):2467-2470

13. Zouboulis CC, Korge B, Akamatsu H, Xia LQ, Schiller S, Gollnick H, Orfanos CE (1991) Effects of 13-cis-retinoic acid, all-trans-retinoic acid, and acitretin on the proliferation, lipid synthesis and keratin expression of cultured human sebocytes in vitro. J Invest Dermatol 96(5):792-797

14. Mukherjee S, Date A, Patravale V, Korting HC, Roeder A, Weindl G (2006) Retinoids in the treatment of skin aging: an overview of clinical efficacy and safety. Clin Interv Aging 1(4):327-348

15. Zhang B, Choi YM, Lee J, An IS, Li L, He C, Dong Y, Bae S (2019) Toll-like receptor 2 plays a critical role in pathogenesis of acne vulgaris. Biomed Dermatol 3:4

16. Kawasaki T, Kawai T (2014) Toll-like receptor signaling pathways. Front Immunol 5:461

17. Chen P, Chen F, Zhou B (2018) Antioxidative, anti-inflammatory and anti-apoptotic effects of ellagic acid in liver and brain of rats treated by D-galactose. Sci Rep 8(1):1465

18. Wang L, Li L, Ran X, Long M, Zhang M, Tao Y, Luo X, Wang Y, Ma X, Halmurati U, Mao X, Ren J (2013) Ellagic acid reduces adipogenesis through inhibition of differentiation-prevention of the induction of Rb phosphorylation in 3T3-L1 adipocytes. Evid Based Complement Alternat Med 2013:287534

19. Lee KH, Jeong ES, Jang G, Na JR, Park S, Kang WS, Kim E, Choi H, Kim JS, Kim S (2020) Unripe Rubus coreanus Miquel extract containing ellagic acid regulates AMPK, SREBP-2, HMGCR, and INSIG-1 signaling and cholesterol metabolism in vitro and in vivo. Nutrients 12(3):610

20. Bae JY, Choi JS, Kang SW, Lee YJ, Park LJ, Kang YH (2010) Dietary compound ellagic acid alleviates skin wrinkle and inflammation induced by UV-B irradiation. Exp dermatol 19(8):e182-190

\section{Publisher's Note}

Springer Nature remains neutral with regard to jurisdictional claims in published maps and institutional affiliations.

\section{Submit your manuscript to a SpringerOpen ${ }^{\circ}$ journal and benefit from:}

- Convenient online submission

- Rigorous peer review

- Open access: articles freely available online

- High visibility within the field

- Retaining the copyright to your article

Submit your next manuscript at $\boldsymbol{\nabla}$ springeropen.com 